\title{
Relations between elliptic modular graphs
}

\author{
Anirban Basu \\ Harish-Chandra Research Institute, HBNI, \\ Chhatnag Road, Jhusi, Prayagraj 211019, India \\ E-mail: anirbanbasu@hri.res.in
}

\begin{abstract}
We consider certain elliptic modular graph functions that arise in the asymptotic expansion around the non-separating node of genus two string invariants that appear in the integrand of the $D^{8} \mathcal{R}^{4}$ interaction in the low momentum expansion of the four graviton amplitude in type II superstring theory. These elliptic modular graphs have links given by the Green function, as well its holomorphic and anti-holomorphic derivatives. Using appropriate auxiliary graphs at various intermediate stages of the analysis, we show that each graph can be expressed solely in terms of graphs with links given only by the Green function and not its derivatives. This results in a reduction in the number of basis elements in the space of elliptic modular graphs.
\end{abstract}

KeYwords: Extended Supersymmetry, Superstrings and Heterotic Strings

ARXIV EPRINT: 2010.08331 


\section{Contents}

1 Introduction 1

2 The elliptic modular graph functions having links involving derivatives of the Green function

3 Relations between the various modular graphs $\quad 6$

3.1 Relation involving $\mathcal{K}_{1}(v) \quad 8$

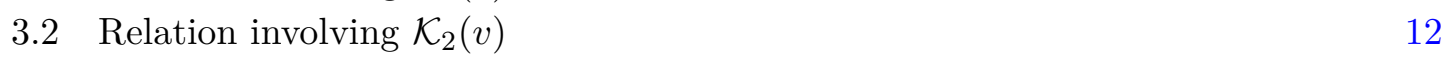

$\begin{array}{lll}3.3 & \text { Relation involving } \mathcal{K}_{3}(v) & 13\end{array}$

$\begin{array}{lll}3.4 & \text { Relation involving } \mathcal{K}_{4}(v) & 15\end{array}$

$\begin{array}{lll}3.5 & \text { Relation involving } \mathcal{K}_{5} & 17\end{array}$

$\begin{array}{lll}3.6 & \text { Some consistency checks } & 18\end{array}$

$\begin{array}{ll}\text { A Some elliptic modular graphs and useful identities } & 19\end{array}$

\section{Introduction}

S-matrix elements contain very useful information about terms in the low energy effective action of superstring theory. In perturbative string theory, they yield terms with coupling dependence $g_{s}^{2 h-2}$ at genus $h$, where $g_{s}$ is the string coupling. Each such contribution at fixed $h$ can be expanded in powers of $\alpha^{\prime}$, the inverse string tension, to yield terms in the effective action that are analytic as well as non-analytic in the external momenta. Focussing on the terms that are analytic in the external momenta, thus we see that the coefficients of the various interactions in the effective action are given by integrals over the moduli space of (super)Riemann surfaces with punctures. These have been explicitly evaluated in various cases for low genera. In such cases, the integrands which depend on the particular string theory under consideration, are given by a sum of terms, each of which has basic building blocks which are referred to as modular graph forms $[1,2]$. These are $\operatorname{Sp}(2 h, \mathbb{Z})$ covariant objects which can be interpreted graphically very usefully. The links of these graphs are given by the conformally invariant Arakelov Green function on the genus $h$ worldsheet or their holomorphic or anti-holomorphic derivatives, while the vertices correspond to the positions of insertions of vertex operators on the worldsheet which are integrated over. Modular graph functions which are $\operatorname{Sp}(2 h, \mathbb{Z})$ invariant are special cases of modular graph forms, and will be the relevant objects for us. We shall consider them in the context of type II superstring theory, though the techniques should generalize to yield information in cases with lesser supersymmetry.

Analysis of these string invariants at genus one that result from the low momentum expansion of one loop amplitudes [3-7] has yielded detailed information about various 
relations among them, as well as played a crucial role in performing the integrals over moduli space [1, 2, 7-20]. Analogous analysis has also been done at genus two [21-30] based on the low momentum expansion of the two loop four and five graviton amplitudes [31-34], though much less has been understood compared to the one loop graphs.

Consider the string invariants that arise in the evaluation of the $D^{8} \mathcal{R}^{4}$ and $D^{6} \mathcal{R}^{5}$ interactions in the low momentum expansion of the four and five graviton amplitudes respectively, at genus two. The asymptotic expansions of these invariants around the nonseparating node yield elliptic modular graph functions $[2,29]$, graphs in which two of the vertices are not integrated over the worldsheet. These graphs depend on $\tau$, the complex structure of the resulting torus at the non-separating node as well as on $p_{a}$ and $p_{b}$, the locations of the two punctures on the genus two Riemann surface which are connected by a long handle, roughly the inverse length of which is the asymptotic expansion parameter. ${ }^{1}$ In fact, using translational invariance on the torus, these graphs depend only on the difference $v=p_{b}-p_{a}$. Thus the two unintegrated vertices are at $v$ and 0 . These generalize the modular graph functions in which all the vertices are integrated over, ${ }^{2}$ and are invariant under the $\mathrm{SL}(2, \mathbb{Z})$ transformation

$$
\tau \rightarrow \frac{a \tau+b}{c \tau+d}, \quad v \rightarrow \frac{v}{c \tau+d},
$$

where $a, b, c, d \in \mathbb{Z}$ and $a d-b c=1$.

Now most of the elliptic modular graph functions that arise in this analysis have links that are given by the Green function on the toroidal worldsheet. However, the ones that arise from the asymptotic expansion of the genus two graphs

$$
\int_{\Sigma_{2}^{2}} \prod_{i=1}^{2} d^{2} z_{i} \mathcal{G}\left(z_{1}, z_{2}\right)^{2} \mu\left(z_{1}\right) \mu\left(z_{2}\right)
$$

and

$$
\int_{\Sigma_{2}^{2}} \prod_{i=1}^{2} d^{2} z_{i} \mathcal{G}\left(z_{1}, z_{2}\right)^{2}\left(z_{1}, \overline{z_{2}}\right)\left(z_{2}, \overline{z_{1}}\right)
$$

are different from this point of view. In fact, (1.2) and (1.3) arise in the low momentum expansion of the four and five graviton amplitude respectively. In these expressions, $\mathcal{G}\left(z_{1}, z_{2}\right)$ is the genus two Arakelov Green function, and

$$
\mu(z)=Y_{I J}^{-1} \omega_{I}(z) \overline{\omega_{J}(z)}, \quad(z, \bar{w})=Y_{I J}^{-1} \omega_{I}(z) \overline{\omega_{J}(w)}
$$

where $Y_{I J}^{-1}=\left(Y^{-1}\right)_{I J}$ and $\omega_{I}=\omega_{I}(z) d z$ is the Abelian differential one form. We have defined $Y_{I J}=(\operatorname{Im} \Omega)_{I J}$, where $\Omega$ is the period matrix. The integrals are over $\Sigma_{2}$, the genus two worldsheet.

Along with elliptic graphs with links given by the Green function, the asymptotic expansion of (1.2) and (1.3) also yields graphs in which the links are given not only by the

\footnotetext{
${ }^{1}$ The expansion yields a Laurent series in this parameter, with the elliptic graphs arising as coefficients in this series.

${ }^{2}$ When the vertices $v$ and 0 are identified, the elliptic modular graphs reduce to modular graphs.
} 
Green function, but also by its holomorphic and anti-holomorphic derivatives. ${ }^{3}$ Thus they yield graphs that are of a qualitatively different kind. ${ }^{4}$ Essentially this happens because the graphs (1.2) and (1.3) form a closed loop on the worldsheet and this involved structure arises from the structure of the Green function in the degeneration limit. It is interesting to analyze if these graphs can be expressed in terms of those that do not have derivatives of Green functions as their links, or if they are genuinely new graphs which add to the number of basis elements in the space of elliptic modular graphs. We shall show that each of these graphs can be expressed solely in terms of those that do not have derivatives of Green functions as their links, leading to a simplification in the structure of the amplitude. It will be interesting to see to what extent this structure survives for elliptic graphs at higher orders in the low momentum expansion.

We begin by giving the details of the various elliptic modular graphs as well as the other modular graphs that are relevant to our analysis. We then show that each of the graphs having derivatives of Green functions as their links can be expressed in terms of those that have only Green functions as their links, which is the main result of the paper. This is done by introducing auxiliary graphs [12-14] at various intermediate stages of the analysis.

\section{The elliptic modular graph functions having links involving derivatives of the Green function}

We first begin by setting up notations and conventions characterizing the torus that is relevant for our analysis. Denoting by $z$ the coordinate on the torus, we have that

$$
-\frac{1}{2} \leq \operatorname{Re} z \leq \frac{1}{2}, \quad 0 \leq \operatorname{Im} z \leq \tau_{2},
$$

where $\tau=\tau_{1}+i \tau_{2}$ is its complex structure. The measure in the various integrals below is given by $d^{2} z=d \operatorname{Re} z d \operatorname{Im} z$. In the integrals over the toroidal worldsheet, we shall denote the worldsheet by $\Sigma$.

In the various graphs, the links involve the scalar Green function $G(z, w)=G(z-w)$ on the toroidal worldsheet, as well as their holomorphic and anti-holomorphic derivatives. The Green function is given by the lattice sum [3, 35]

$$
G(z)=\frac{1}{\pi} \sum_{(m, n) \neq(0,0)} \frac{\tau_{2}}{|m \tau+n|^{2}} e^{\pi[\bar{z}(m \tau+n)-z(m \bar{\tau}+n)] / \tau_{2}} .
$$

It is modular invariant and doubly periodic on the torus. Since it is single valued, we can freely integrate by parts and neglect total derivatives which is very useful in our calculations.

\footnotetext{
${ }^{3}$ In fact, the two graphs (1.2) and (1.3) are related [29] by a non-trivial algebraic identity which also involves other graphs, none of which contain elliptic graphs in their asymptotic expansion that involve derivatives of the Green function. Thus to consider graphs involving derivatives of the Green function, it is enough to either consider (1.2) or (1.3).

${ }^{4}$ This situation is somewhat analogous to modular graphs that arise in the low momentum expansion of the five graviton amplitude at genus one that yield the $D^{8} \mathcal{R}^{5}$ and $D^{10} \mathcal{R}^{5}$ interactions in the type IIB theory, where some of them involve derivatives of the Green function [6]. However, all these graphs can be expressed in terms of graphs not involving derivatives of the Green function [14], hence simplifying the structure of the amplitude.
} 
Also from the definition (2.2) of the Green function, it follows that

$$
\int_{\Sigma} d^{2} z G(z, w)=0
$$

Thus there can be no graphs where a single link ends on an integrated vertex, a relation which we often use.

The Green function satisfies the equations

$$
\begin{aligned}
& \bar{\partial}_{w} \partial_{z} G(z, w)=\pi \delta^{2}(z-w)-\frac{\pi}{\tau_{2}} \\
& \bar{\partial}_{z} \partial_{z} G(z, w)=-\pi \delta^{2}(z-w)+\frac{\pi}{\tau_{2}}
\end{aligned}
$$

where the Dirac delta function is normalized such that $\int_{\Sigma} d^{2} z \delta^{2}(z)=1$.

We now list the elliptic modular graph functions that not only have Green functions as their links, but also its holomorphic and anti-holomorphic derivatives [27]. Apart from the complex structure $\tau$, they are also functions of the complex parameter $v=p_{b}-p_{a}$, where $p_{a}$ and $p_{b}$ are the locations of the punctures on the genus two worldsheet which are connected by a long handle at the non-separating node.

To start with, we define

$$
\mathcal{K}_{1}(v)=\frac{\tau_{2}^{2}}{\pi^{2}} \int_{\Sigma^{2}} \frac{d^{2} z}{\tau_{2}} \frac{d^{2} w}{\tau_{2}} \partial_{z} G\left(z, p_{a}\right) \bar{\partial}_{z} G\left(z, p_{b}\right) G(z, w)^{2} \partial_{w} G\left(w, p_{a}\right) \bar{\partial}_{w} G\left(w, p_{b}\right),
$$

as well as

$$
\mathcal{K}_{2}(v)=\frac{\tau_{2}^{2}}{\pi^{2}} \int_{\Sigma^{2}} \frac{d^{2} z}{\tau_{2}} \frac{d^{2} w}{\tau_{2}} \partial_{z} G\left(z, p_{a}\right) \bar{\partial}_{z} G\left(z, p_{b}\right) G(z, w)^{2} \partial_{w} G\left(w, p_{b}\right) \bar{\partial}_{w} G\left(w, p_{a}\right)
$$

which has the same topology as $\mathcal{K}_{1}$ but has two derivatives interchanged. Both these graphs are three loop graphs on the toroidal worldsheet. ${ }^{5}$

Apart from these graphs, we also have other multiloop graphs. One of them is defined by

$$
\widetilde{\mathcal{K}}_{3}(v)=\frac{\tau_{2}^{2}}{\pi^{2}} \int_{\Sigma^{2}} \frac{d^{2} z}{\tau_{2}} \frac{d^{2} w}{\tau_{2}}\left|\partial_{z} G\left(z, p_{a}\right)\right|^{2} \partial_{w} G\left(w, p_{a}\right) \bar{\partial}_{w} G\left(w, p_{b}\right)\left[G(z, w)^{2}-G\left(w, p_{a}\right)^{2}\right] .
$$

This graph is complex and in the amplitude we thus have to consider $\widetilde{\mathcal{K}}_{3}(v)+$ c.c..

The remaining graphs are defined by

$$
\begin{aligned}
\widetilde{\mathcal{K}}_{4}(v)=\frac{\tau_{2}^{2}}{\pi^{2}} \int_{\Sigma^{2}} \frac{d^{2} z}{\tau_{2}} \frac{d^{2} w}{\tau_{2}}\left|\partial_{z} G\left(z, p_{a}\right)\right|^{2}\left|\partial_{w} G\left(w, p_{b}\right)\right|^{2}[ & G(z, w)^{2}-G\left(w, p_{a}\right)^{2} \\
& \left.-G\left(z, p_{b}\right)^{2}+G\left(p_{a}, p_{b}\right)^{2}\right],
\end{aligned}
$$

and

$$
\widetilde{\mathcal{K}}_{5}=\frac{\tau_{2}}{\pi} \int_{\Sigma} \frac{d^{2} z}{\tau_{2}}\left|\partial_{z} G(z)\right|^{2}[W(z)-4 \zeta(3)]
$$

\footnotetext{
${ }^{5}$ From now onwards loops refer to the loops in the graph, and not to string loops. All the graphs are at genus one.
} 
where

$$
W(z)=\frac{\tau_{2}}{\pi} \int_{\Sigma} \frac{d^{2} w}{\tau_{2}}\left|\partial_{w} G(w)\right|^{2}(G(z, w)-G(z))(G(z, w)-G(w)) .
$$

Note that $\widetilde{\mathcal{K}}_{5}$ is independent of $v$, and hence is a modular graph, but not an elliptic modular graph.

For the sake of brevity, from now onwards, we shall simply refer to all graphs as modular graphs.

In analyzing these graphs, at certain intermediate steps we obtain expressions involving $G(z, z)$, the Green function at coincident points. We simply set $G(z, z)=0$ in such situations, which is the natural and standard thing to do. ${ }^{6}$ This is because in defining the graphs the vertex operators are never allowed to coincide, as such colliding vertex operators produce another local vertex operator at that point using the operator product expansion. Hence coincident Green functions are not in the moduli space of these graphs, and can be consistently ignored.

Thus we have that

$$
\int_{\Sigma} d^{2} z\left|\partial_{z} G(z, w)\right|^{2}=0
$$

where we have integrated by parts, and used (2.4) and (2.3). This allows us the simplify the expressions for the various graphs we have listed above.

We see that $\mathcal{K}_{1}(v)$ and $\mathcal{K}_{2}(v)$ are as given in (2.5) and (2.6) respectively. On the other hand, $\widetilde{\mathcal{K}}_{3}(v)$ in $(2.7)$ reduces to the three loop graph

$$
\mathcal{K}_{3}(v)=\frac{\tau_{2}^{2}}{\pi^{2}} \int_{\Sigma^{2}} \frac{d^{2} z}{\tau_{2}} \frac{d^{2} w}{\tau_{2}}\left|\partial_{z} G\left(z, p_{a}\right)\right|^{2} \partial_{w} G\left(w, p_{a}\right) \bar{\partial}_{w} G\left(w, p_{b}\right) G(z, w)^{2},
$$

and so we shall consider $\mathcal{K}_{3}(v)+$ c.c..

Also $\widetilde{\mathcal{K}}_{4}(v)$ in $(2.8)$ reduces to a three loop graph

$$
\mathcal{K}_{4}(v)=\frac{\tau_{2}^{2}}{\pi^{2}} \int_{\Sigma^{2}} \frac{d^{2} z}{\tau_{2}} \frac{d^{2} w}{\tau_{2}}\left|\partial_{z} G\left(z, p_{a}\right)\right|^{2}\left|\partial_{w} G\left(w, p_{b}\right)\right|^{2} G(z, w)^{2}
$$

with distinct topology compared to $\mathcal{K}_{3}(v)$.

Finally, $\widetilde{\mathcal{K}}_{5}$ in $(2.9)$ reduces to a four loop graph given by

$$
\mathcal{K}_{5}=\frac{\tau_{2}^{2}}{\pi^{2}} \int_{\Sigma^{2}} \frac{d^{2} z}{\tau_{2}} \frac{d^{2} w}{\tau_{2}}\left|\partial_{z} G(z)\right|^{2}\left|\partial_{w} G(w)\right|^{2}(G(z, w)-G(z))(G(z, w)-G(w)),
$$

since only the contribution involving $W(z)$ survives. Thus among the graphs we consider, this is the only graph with more than one term. We shall depict all these graphs diagrammatically later on.

We shall need the expression for the $\mathrm{SL}(2, \mathbb{Z})$ invariant Laplacian involving derivatives with respect to the complex structure given by

$$
\Delta_{\tau}=4 \tau_{2}^{2} \frac{\partial^{2}}{\partial \tau \partial \bar{\tau}}
$$

\footnotetext{
${ }^{6}$ See [6] for example, for a recent discussion.
} 


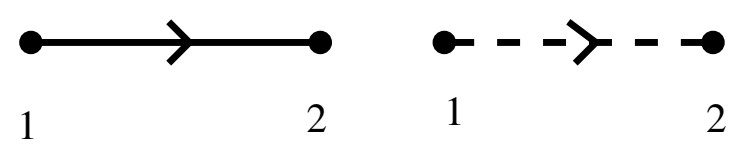

i $\quad$ ii

Figure 1. (i) $\partial_{z_{2}} G\left(z_{1}, z_{2}\right)=-\partial_{z_{1}} G\left(z_{1}, z_{2}\right)$, (ii) $\bar{\partial}_{z_{2}} G\left(z_{1}, z_{2}\right)=-\bar{\partial}_{z_{1}} G\left(z_{1}, z_{2}\right)$.

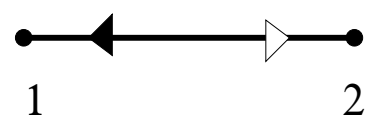

Figure 2. $\bar{\partial}_{z_{1}} \partial_{z_{2}} G\left(z_{1}, z_{2}\right)$.

We shall also find useful the $\mathrm{SL}(2, \mathbb{Z})$ invariant Laplacian involving derivatives with respect to the complex parameter $v$ on the torus given by

$$
\Delta_{v}=4 \tau_{2} \frac{\partial^{2}}{\partial v \partial \bar{v}}
$$

In the various graphs, the unintegrated vertices $v$ and 0 can be interchanged which we shall use implicitly, which simply follows from the properties of the Green function (2.2).

In our analysis, it is very useful to diagrammatically depict these graphs. While the Green function along a link is given by a solid line, holomorphic and anti-holomorphic derivatives of Green functions are depicted by figure 1. Such derivatives appearing in the same link is depicted by figure 2 .

\section{Relations between the various modular graphs}

Apart from the graphs $\mathcal{K}_{i}(v)(i=1, \ldots, 5)$ mentioned above, several other graphs appear in our analysis which we now list. The relevant elliptic modular graphs are given in figure 3. The unintegrated vertices of such graphs (denoted by $v$ and 0 ) are always depicted in the figures, while all other vertices are integrated over.

From now onwards, for the sake of brevity we shall refer to $\int_{\Sigma} d^{2} z / \tau_{2}$ simply as $\int_{z}$ in the various expressions.

Some of the graphs in figure 3 are defined by [27]

$$
D_{l}^{(k)}(v)=\int_{z} G^{k}(v, z) G(z)^{l-k}=D_{l}^{(l-k)}(v)
$$

for $l \geq 2$ and $k \leq l$. Some of the others are given by the iterated Green functions $G_{k}(v)$ $(k \geq 1)$ defined recursively by

$$
G_{k+1}(v)=\int_{z} G(v, z) G_{k}(z)
$$




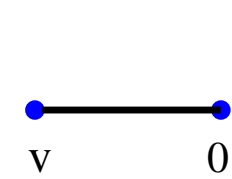

(i)

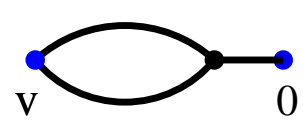

(iv)

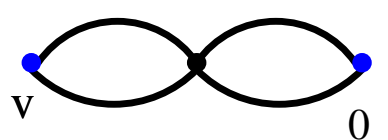

(vii)

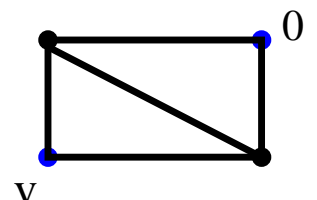

(x)

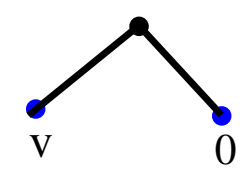

(ii)

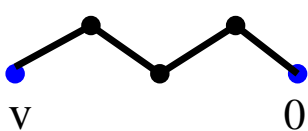

(v)

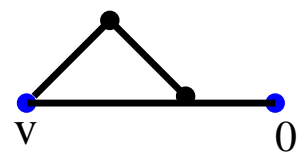

(viii)

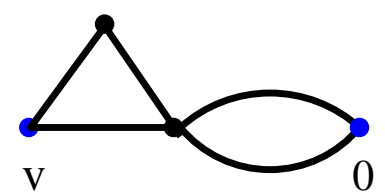

(xi)

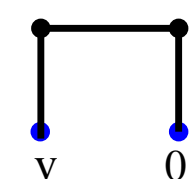

(iii)

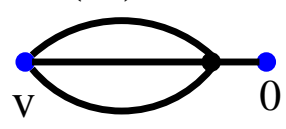

(vi)

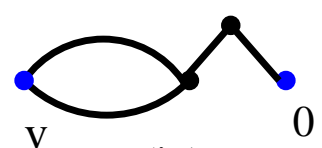

(ix)

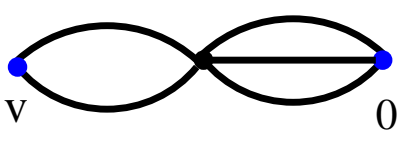

(xii)

Figure 3. The elliptic modular graphs (i) $G(v)$, (ii) $G_{2}(v)$, (iii) $G_{3}(v)$, (iv) $D_{3}^{(1)}(v)$, (v) $G_{4}(v)$, (vi) $D_{4}^{(1)}(v)$, (vii) $D_{4}^{(2)}(v)$, (viii) $D_{4}^{(1,2)}(v)$, (ix) $D_{4}^{(1,1,2)}(v),(\mathrm{x}) D_{5}^{(2,1,2)}(v)$, (xi) $D_{5}^{(1,2,2)}(v)$, (xii) $D_{5}^{(2)}(v)$.

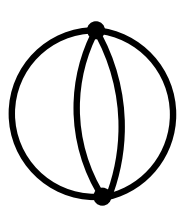

(i)

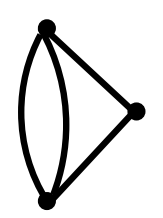

(ii)

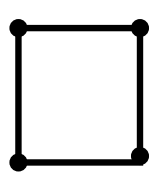

(iii)

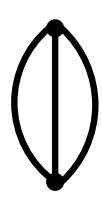

(iv)

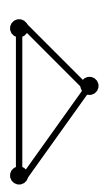

(v)

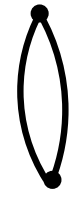

(vi)

Figure 4. (i) $D_{4}$, (ii) $C_{1,1,2}$, (iii) $E_{4}$, (iv) $D_{3}$, (v) $E_{3}$, (vi) $E_{2}$.

where $G_{1}(z)=G(z)$, the Green function. The remaining graphs are defined by the integrals

$$
\begin{aligned}
D_{4}^{(1,2)}(v) & =\int_{w z} G(w) G(w, v) G(z, v) G(w, z), \\
D_{4}^{(1,1,2)}(v) & =\int_{w z} G(w) G(z, v)^{2} G(w, z), \\
D_{5}^{(2,1,2)}(v) & =\int_{w z} G(w) G(z) G(w, v) G(z, v) G(w, z), \\
D_{5}^{(1,2,2)}(v) & =\int_{w z} G(w)^{2} G(w, v) G(z, v) G(w, z) .
\end{aligned}
$$

We next mention the various modular graphs that arise in our analysis which are given in figure 4.

These can be obtained from some of the elliptic modular graphs mentioned above by identifying $v$ and 0 . Thus we have that $D_{l}^{(k)}(0)=D_{l}$ for $l \geq 2, k \leq l$, and $G_{k+1}(0)=E_{k+1}$ 


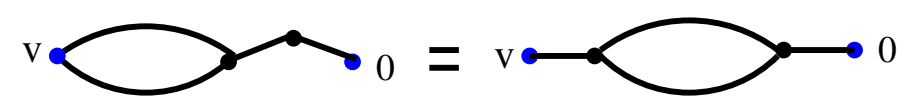

Figure 5. A relation between elliptic graphs.

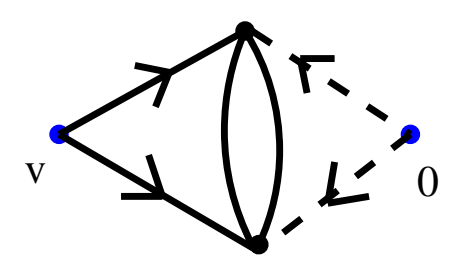

Figure 6. $\pi^{2} \mathcal{K}_{1}(v)$.

for $k \geq 1$. We also have that

$$
D_{4}^{(1,2)}(0)=D_{4}^{(1,1,2)}(0)=C_{1,1,2}
$$

We also often use the relation between the elliptic graphs given in figure 5 [36], where the graph on the right hand side is given by

$$
\int_{w z} G(z, v) G(w) G(w, z)^{2} .
$$

We now proceed to obtain the relation between each of the graphs $\mathcal{K}_{i}(v)(i=1, \ldots, 5)$ and the other graphs given in figures 3 and 4 . Note that all the links in all the graphs in figures 3 and 4 involve only the Green function, and not its derivatives.

Thus schematically the aim is to express each of the graphs $\mathcal{K}_{i}(v)$ in terms of graphs having no derivatives of Green functions as the links. The strategy we shall follow starting from $\mathcal{K}_{i}(v)$ is to use the single valuedness of the Green function (2.2) and integrate by parts, and use (2.4) to remove the derivatives. Now this does not always work in a straightforward way in removing derivatives, as will be evident in the manipulations below. To proceed in such cases, we shall introduce auxiliary graphs at various intermediate stages of the analysis. To summarize, at the end we shall express each $\mathcal{K}_{i}(v)$ in terms of graphs that appear in figures 3 and 4 .

\subsection{Relation involving $\mathcal{K}_{1}(v)$}

We first consider the relation involving the graph $\mathcal{K}_{1}(v)$ defined by $(2.5)$.

Thus we have that

$$
\mathcal{K}_{1}(v)=\frac{\tau_{2}^{2}}{\pi^{2}} \int_{w z} \partial_{z} G(z, v) \bar{\partial}_{z} G(z) G(z, w)^{2} \partial_{w} G(w, v) \bar{\partial}_{w} G(w)
$$

as depicted by figure 6 .

Integrating appropriately by parts and using (2.4), we get that

$$
\mathcal{K}_{1}(v)=\frac{4}{3} D_{3} G(v)-\frac{G(v)^{4}}{3}-D_{4}^{(1,1,2)}(v)-2 F_{1}(v)-\left(F_{2}(v)+c . c .\right)+2 H_{1}(v),
$$




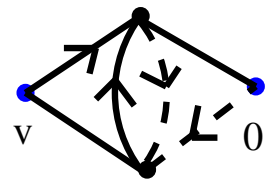

(i)

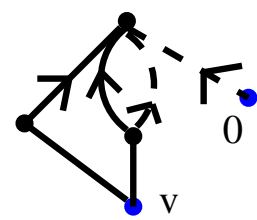

(iv)

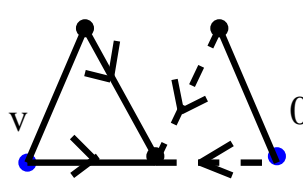

(ii)

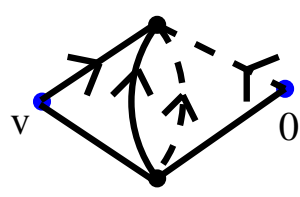

(v)

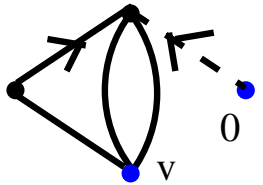

(iii)

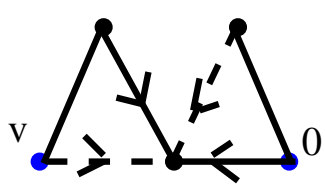

(vi)

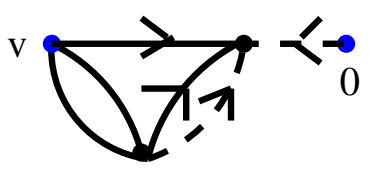

(vii)

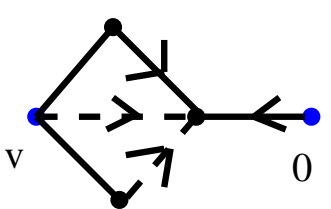

(ix)

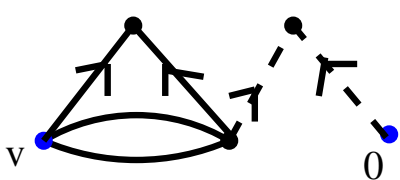

(viii)

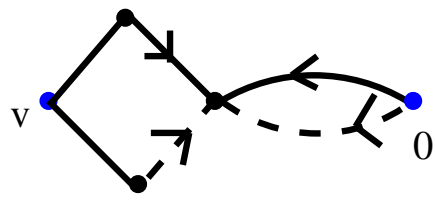

(X)

Figure 7. The graphs (i) $\pi^{2} H_{1}(v)$, (ii) $\pi^{2} H_{2}(v)$, (iii) $\pi H_{3}(v)$, (iv) $\pi^{2} H_{4}(v)$, (v) $\pi^{2} H_{5}(v)$, (vi) $\pi^{2} H_{6}(v)$, (vii) $\pi^{2} H_{7}(v)$, (viii) $\pi^{2} H_{8}(v)$, (ix) $\pi^{2} H_{9}(v)$, (x) $\pi^{2} H_{10}(v)$.

where

$$
H_{1}(v)=\frac{\tau_{2}^{2}}{\pi^{2}} \int_{w z} \partial_{z} G(z, w) \bar{\partial}_{w} G(z, w) \partial_{z} G(z, v) \bar{\partial}_{w} G(w) G(w, v) G(z)
$$

as depicted in figure 7 . The graphs $F_{1}(v)$ and $F_{2}(v)$ are defined by $(\mathrm{A} .1)$ and given in figure 13 .

Thus using (A.4), we see that all the terms on the right hand side of (3.7) except the one involving $H_{1}(v)$ are expressible in terms of graphs having only the Green function as the links.

Now let us consider the term $H_{1}(v)$. To analyze it, we shall introduce appropriate auxiliary graphs [12-14]. In general, an auxiliary graph is a graph which trivially yields the desired graph using (2.4). However, it can be evaluated independently by integrating by parts and using (2.4) such that all the derivatives are removed. Thus equating the two distinct ways of evaluating the auxiliary graph, we get an expression in which the derivatives in the desired graph are completely removed, in the sense that it is expressed in terms of graphs without derivatives of the Green function as the links. Often this complete reduction requires introducing more than one auxiliary graph at various intermediate stages of the analysis. 


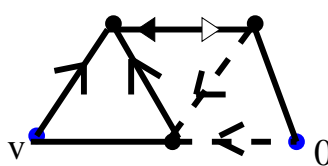

(i)

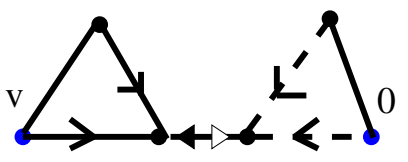

(ii)

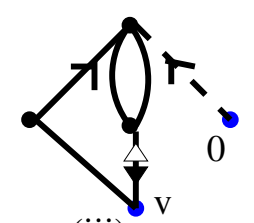

(iii)

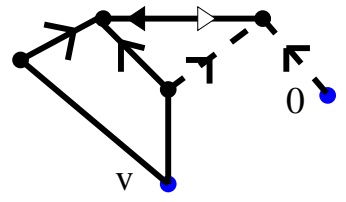

(iv)

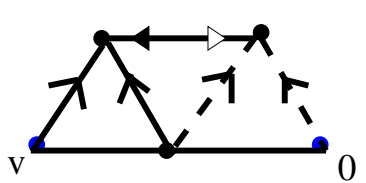

(v)

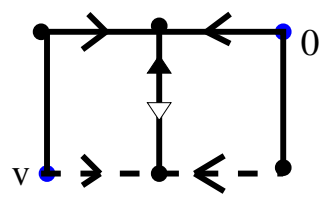

(vi)

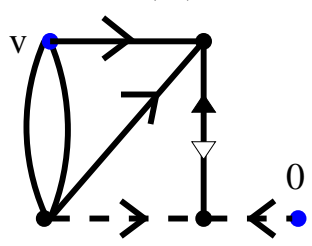

(vii)

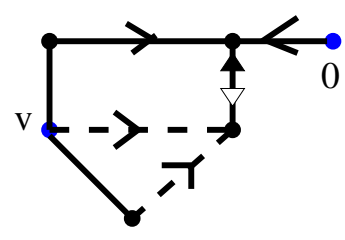

(viii)

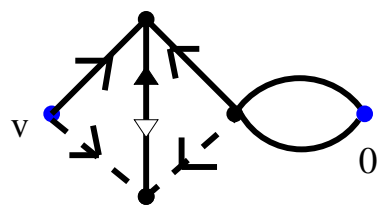

(ix)

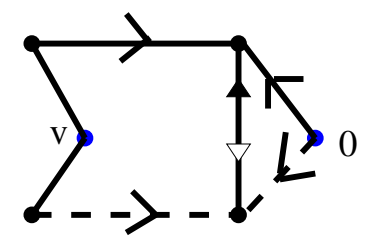

(x)

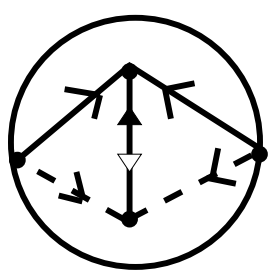

(xi)

Figure 8. The auxiliary graphs (i) $\pi^{3} A_{1}(v)$, (ii) $\pi^{3} A_{2}(v)$, (iii) $\pi^{2} A_{3}(v)$, (iv) $\pi^{3} A_{4}(v)$, (v) $\pi^{3} A_{5}(v)$, (vi) $\pi^{3} A_{6}(v)$, (vii) $\pi^{3} A_{7}(v)$, (viii) $\pi^{3} A_{8}(v)$, (ix) $\pi^{3} A_{9}(v)$, (x) $\pi^{3} A_{10}(v)$, (xi) $\pi^{3} A_{11}(v)$.

To analyze $H_{1}(v)$, we begin with the auxiliary graph $A_{1}(v)+$ c.c., where

$$
A_{1}(v)=\frac{\tau_{2}^{3}}{\pi^{3}} \int_{x w z} \partial_{w} \bar{\partial}_{z} G(z, w) \partial_{z} G(z, v) \partial_{z} G(x, z) \bar{\partial}_{x} G(x, w) \bar{\partial}_{x} G(x) G(w) G(x, v),
$$

as given in figure 8. It is evaluated trivially using (2.4) for the link having a $\partial$ as well as a $\bar{\partial}$ acting on the Green function, and alternatively by moving the derivatives around the circuit and using (2.4). This will be the strategy we shall always follow.

This leads to

$$
\begin{aligned}
H_{1}(v)= & \frac{D_{4}^{(1)}(v)}{2}+\frac{E_{2}^{2}}{4}+D_{4}^{(1,2)}(v)-\frac{D_{4}^{(1,1,2)}(v)}{2}-\frac{D_{4}^{(2)}(v)}{2}+\frac{G_{2}(v)^{2}}{2}+\frac{G(v)^{4}}{4} \\
& +F_{1}(v)+\frac{1}{2}\left(F_{3}(v)+c . c .\right)-\frac{1}{4}\left(F_{4}(v)+c . c .\right)-\frac{1}{2}\left(F_{5}(v)+c . c .\right) \\
& -\frac{1}{4}\left(F_{6}(v)+c . c .\right)+\frac{1}{2}\left(\frac{\tau_{2}}{\pi} \partial_{v} G_{2}(v) \bar{\partial}_{v} G_{3}(v)+c . c .\right)+H_{2}(v)-\frac{1}{4}\left(H_{3}(v)+c . c .\right),
\end{aligned}
$$


where we define

$$
\begin{aligned}
& H_{2}(v)=\frac{\tau_{2}^{2}}{\pi^{2}} \int_{w x z} G(z, v) G(w) \partial_{x} G(x, z) \partial_{x} G(x, v) \bar{\partial}_{x} G(x, w) \bar{\partial}_{x} G(x), \\
& H_{3}(v)=\frac{\tau_{2}}{\pi} \int_{w z} G(z, v)^{2} G(w, v) \partial_{z} G(w, z) \bar{\partial}_{z} G(z)
\end{aligned}
$$

as depicted by figure 7. Also the graphs $F_{i}(v)$ are defined by (A.1) and depicted by figure 13 .

From the relations in (A.4) and (A.12), we see that apart from the terms involving $H_{2}(v)$ and $H_{3}(v)$ on the right hand side of (3.10), all the other terms can be expressed in terms of graphs without derivatives of the Green function as the links. To analyze $\mathrm{H}_{2}(v)$, we consider the auxiliary graph

$$
A_{2}(v)=\frac{\tau_{2}^{3}}{\pi^{3}} \int_{w x y z} G(z, v) G(w) \bar{\partial}_{x} \partial_{y} G(x, y) \partial_{x} G(x, z) \partial_{x} G(x, v) \bar{\partial}_{y} G(y) \bar{\partial}_{y} G(w, y),
$$

as given in figure 8 , which leads to

$$
H_{2}(v)=Q_{1} Q_{1}^{*}-D_{4}^{(1,1,2)}(v)+\frac{D_{4}^{(2)}(v)}{4}-\frac{E_{2}^{2}}{4}+G_{4}(v),
$$

where $Q_{1}$ is defined by (A.2). From (A.10) we see that $H_{2}(v)$ is expressible in terms of graphs without derivatives of the Green function as its links.

Next to analyze $H_{3}(v)+c . c$, we consider the auxiliary graph ${ }^{7}$

$$
A_{3}(v)=\frac{\tau_{2}^{2}}{\pi^{2}} \int_{w x z} G(x, v) G(z, w)^{2} \partial_{z} G(x, z) \bar{\partial}_{z} G(z) \partial_{w} \bar{\partial}_{v} G(w, v),
$$

depicted by figure 8 , which leads to the relation

$$
H_{3}(v)=E_{2} G_{2}(v)-G_{2}(v)^{2}+E_{4}-2 H_{4}(v)
$$

where the graph $H_{4}(v)$ is defined by

$$
H_{4}(v)=\frac{\tau_{2}^{2}}{\pi^{2}} \int_{w x z} G(x, v) G(w, v) \partial_{z} G(x, z) \partial_{z} G(w, z) \bar{\partial}_{z} G(w, z) \bar{\partial}_{z} G(z)
$$

as depicted by figure 7 . To analyze $H_{4}(v)$, we consider the auxiliary graph $A_{4}(v)$ defined by

$$
A_{4}(v)=\frac{\tau_{2}^{3}}{\pi^{3}} \int_{w x y z} G(y, v) G(x, v) \partial_{w} G(w, y) \partial_{w} G(w, x) \bar{\partial}_{z} G(x, z) \bar{\partial}_{z} G(z) \bar{\partial}_{w} \partial_{z} G(w, z)
$$

depicted by figure 8 . This leads to the relation

$$
\begin{aligned}
H_{4}(v)= & G_{4}(v)+\frac{3 E_{4}}{2}-D_{4}^{(1,2)}(v)-\frac{D_{4}^{(2)}(v)}{2}-\frac{E_{2}^{2}}{2}+\frac{C_{1,1,2}}{2}-\frac{1}{2} G(v) D_{3}^{(1)}(v) \\
& -\frac{\tau_{2}}{\pi} \bar{\partial}_{v} G_{2}(v) \partial_{v} G_{3}(v)+\frac{F_{2}(v)}{2}-F_{3}(v)+\frac{F_{6}(v)}{2}+F_{7}(v) .
\end{aligned}
$$

\footnotetext{
${ }^{7}$ This analysis is similar to that in [17].
} 


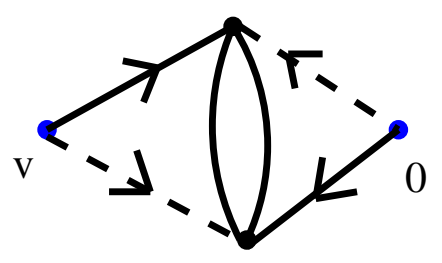

Figure 9. $\pi^{2} \mathcal{K}_{2}(v)$.

Using the definitions in (A.1), and the relations (A.4) and (A.12) we see that the we have expressed $H_{3}(v)$ in terms of graphs without derivatives of the Green function as their links.

Thus from the various relations above, we see that $\mathcal{K}_{1}(v)$ is expressible in terms of the modular graphs given in figures 3 and 4 , in which the links are given by the Green function and not its derivatives. In fact, adding the various contributions, we get that

$$
\begin{aligned}
\mathcal{K}_{1}(v)= & \frac{1}{4}\left(\Delta_{\tau}-8\right) E_{2}^{2}+2 E_{4}+4 G_{4}(v)+G_{2}(v)^{2}-\frac{D_{4}^{(2)}(v)}{2} \\
& -2 D_{4}^{(1,1,2)}(v)-\frac{2}{3} D_{3} G(v)+\frac{G(v)^{4}}{6} .
\end{aligned}
$$

We now analyze the remaining graphs $\mathcal{K}_{i}(v)$ where the basic strategy is the same, and hence we shall be somewhat brief. We shall say that a graph has "simplified", by which we shall always mean that it is expressible in terms of graphs where the links involve only the Green function.

\subsection{Relation involving $\mathcal{K}_{2}(v)$}

We next consider the graph $\mathcal{K}_{2}(v)$ defined by (2.6) leading to

$$
\mathcal{K}_{2}(v)=\frac{\tau_{2}^{2}}{\pi^{2}} \int_{w z} \partial_{z} G(z, v) \bar{\partial}_{z} G(z) G(z, w)^{2} \partial_{w} G(w) \bar{\partial}_{w} G(w, v),
$$

as depicted in figure 9 .

This gives us that

$$
\begin{aligned}
\mathcal{K}_{2}(v)= & \frac{2 G(v)^{4}}{3}-\frac{2}{3} D_{3} G(v)-2 D_{4}^{(1,2)}(v)+2 G(v) D_{3}^{(1)}(v)-D_{4}^{(2)}(v) \\
& +G_{2}(v)^{2}-\left(F_{2}(v)+\text { c.c. }\right)-\mathcal{K}_{1}(v)+2 H_{5}(v)
\end{aligned}
$$

where $H_{5}(v)$ is defined by

$$
H_{5}(v)=\frac{\tau_{2}^{2}}{\pi^{2}} \int_{w z} G(w, v) G(w) \partial_{z} G(z, v) \partial_{z} G(z, w) \bar{\partial}_{z} G(z, w) \bar{\partial}_{z} G(z)
$$

as given in figure 7. Thus using (A.4) and (3.19) we see that apart from the term involving $H_{5}(v)$, the right hand side of (3.21) has simplified. Now to simplify $H_{5}(v)$, we start with the auxiliary graph $A_{5}(v)$ defined by

$$
A_{5}(v)=\frac{\tau_{2}^{3}}{\pi^{3}} \int_{w x z} G(x) G(x, v) \partial_{z} G(z, v) \partial_{z} G(x, z) \bar{\partial}_{w} G(w, x) \bar{\partial}_{w} G(w) \bar{\partial}_{z} \partial_{w} G(w, z)
$$


as depicted by figure 8 . This leads to the expression

$$
\begin{aligned}
H_{5}(v)= & -\frac{1}{2} G(v) D_{3}^{(1)}(v)+\frac{2 F_{8}(v)}{3}-\frac{1}{2} G(v) F_{9}(v)-\frac{D_{4}^{(1)}(v)}{3}+2 D_{4}^{(1,2)}(v) \\
& +E_{2} G_{2}(v)+G_{2}(v)^{2}-D_{4}^{(2)}(v)+\left(F_{3}(v)+c . c .\right) \\
& +\frac{1}{2}\left(H_{3}(v)+\text { c.c. }\right)-\frac{1}{2}\left(\frac{\tau_{2}}{\pi} \partial_{v} G_{2}(v) \bar{\partial}_{v} D_{3}^{(1)}(v)+\text { c.c. }\right)+H_{2}(v)+H_{6}(v),
\end{aligned}
$$

where

$$
H_{6}(v)=\frac{\tau_{2}^{2}}{\pi^{2}} \int_{w x z} G(z, v) G(w) \partial_{x} G(x, z) \bar{\partial}_{x} G(x, v) \partial_{x} G(x) \bar{\partial}_{x} G(w, x)
$$

as depicted by figure 7, and the graphs $F_{i}(v)$ are defined by (A.1). Thus using (A.4), (A.12) and the expressions involving $H_{2}(v)$ and $H_{3}(v)$ in the analysis of $\mathcal{K}_{1}(v)$, we see that all the terms in the right hand side of (3.24) have simplified, except the one involving $H_{6}(v)$.

To simplify $H_{6}(v)$, we consider the auxiliary graph $A_{6}(v)$ defined by

$$
A_{6}(v)=\frac{\tau_{2}^{3}}{\pi^{3}} \int_{w x y z} G(x, v) G(y) \partial_{z} G(x, z) \partial_{z} G(z) \bar{\partial}_{w} G(w, v) \bar{\partial}_{w} G(w, y) \bar{\partial}_{z} \partial_{w} G(w, z),
$$

as given in figure 8 , which leads to the relation

$$
\begin{aligned}
H_{6}(v)= & Q_{2}(v) Q_{2}^{*}(v)+G_{4}(v)+F_{1}(v)-\left(F_{10}(v)+c . c .\right) \\
& +\left(\frac{\tau_{2}}{\pi} \partial_{v} G_{2}(v) \bar{\partial}_{v} D_{3}^{(1)}(v)+c . c .\right)-\frac{\tau_{2}}{\pi} G(v) \partial_{v} G_{2}(v) \bar{\partial}_{v} G_{2}(v) .
\end{aligned}
$$

Thus using (A.2) and the relations (A.4), (A.10) and (A.12), we see that $H_{6}(v)$ has simplified. Adding the various contributions, we get that

$$
\begin{aligned}
\mathcal{K}_{2}(v)= & -\frac{1}{4 \pi} \Delta_{v}\left(D_{5}^{(2,1,2)}(v)+D_{5}^{(1,2,2)}(v)-\frac{2 D_{5}^{(2)}(v)}{3}-2 G_{2}(v) G_{3}(v)-G_{2}(v) D_{3}^{(1)}(v)\right) \\
& -\frac{1}{4 \pi} G(v) \Delta_{v}\left(G_{2}(v)^{2}+\frac{D_{4}^{(2)}(v)}{2}\right)+\frac{1}{4}\left(\Delta_{\tau}+2\right) E_{2}^{2}+\frac{1}{4}\left(\Delta_{\tau}+12\right) G_{2}(v)^{2}+10 D_{4}^{(1,2)}(v) \\
& -\frac{5}{2} D_{4}^{(2)}(v)-3 E_{2} G_{2}(v)+5 D_{4}^{(1,1,2)}(v)+6\left(G_{4}(v)-E_{4}\right)+G(v)\left(\frac{2 G(v)^{3}}{3}-\frac{8 D_{3}}{3}\right. \\
& \left.+D_{3}^{(1)}(v)-E_{2} G(v)+4 G_{3}(v)-2 E_{3}-G(v) G_{2}(v)\right)-\mathcal{K}_{1}(v)
\end{aligned}
$$

and hence $\mathcal{K}_{2}(v)$ has simplified. ${ }^{8}$

\subsection{Relation involving $\mathcal{K}_{3}(v)$}

We next consider the graph $\mathcal{K}_{3}(v)$ defined by $(2.12)$ which we write as

$$
\mathcal{K}_{3}(v)=\frac{\tau_{2}^{2}}{\pi^{2}} \int_{w z}\left|\partial_{z} G(z, v)\right|^{2} \partial_{w} G(w, v) \bar{\partial}_{w} G(w) G(z, w)^{2}
$$

as depicted by figure 10 .

\footnotetext{
${ }^{8}$ In fact, it is only the combination $\mathcal{K}_{1}(v)+\mathcal{K}_{2}(v)$ that arises in the asmyptotic expansion of the $D^{8} \mathcal{R}^{4}$ string invariants [27], which is directly given by (3.28).
} 


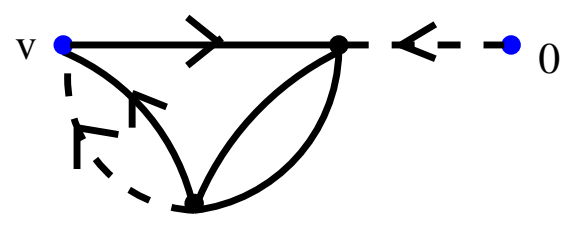

Figure 10. $\pi^{2} \mathcal{K}_{3}(v)$.

Proceeding to simplify it, we get that

$$
\mathcal{K}_{3}(v)=D_{3} G(v)-D_{4}^{(1,1,2)}(v)-F_{2}(v)-F_{4}(v)+H_{7}(v),
$$

where $H_{7}(v)$ is defined by

$$
H_{7}(v)=\frac{\tau_{2}^{2}}{\pi^{2}} \int_{w z} G(w, v)^{2} \partial_{z} G(w, z) \partial_{z} G(z, v) \bar{\partial}_{z} G(w, z) \bar{\partial}_{z} G(z)
$$

as depicted by figure 7. Using (A.4) we see that this is the only term on the right hand side of (3.30) which has not simplified.

To simplify it, we consider the auxiliary graph $A_{7}(v)$ defined by

$$
A_{7}(v)=\frac{\tau_{2}^{3}}{\pi^{3}} \int_{w x z} G(w, v)^{2} \partial_{z} G(z, v) \partial_{z} G(w, z) \bar{\partial}_{x} G(w, x) \bar{\partial}_{x} G(x) \bar{\partial}_{z} \partial_{x} G(x, z)
$$

as given in figure 8 . This yields the expression

$$
\begin{aligned}
H_{7}(v)= & \frac{G(v)^{4}}{12}-\frac{D_{4}}{12}+C_{1,1,2}+E_{2} G_{2}(v)+E_{2}^{2}+D_{4}^{(1,1,2)}(v)-\frac{1}{3} D_{3} G(v) \\
& -H_{3}(v)-F_{6}(v)+\frac{F_{8}(v)}{3}-\frac{\tau_{2}}{\pi} \partial_{v} G_{2}(v) \bar{\partial}_{v} D_{3}^{(1)}(v)+H_{8}(v),
\end{aligned}
$$

where we define

$$
H_{8}(v)=\frac{\tau_{2}^{2}}{\pi^{2}} \int_{w x z} G(w, v)^{2} \partial_{z} G(z, v) \partial_{z} G(w, z) \bar{\partial}_{x} G(w, x) \bar{\partial}_{x} G(x)
$$

as given in figure 7. Based on (A.4), (A.12) and the previous analysis of $H_{3}(v)$, this is the only term which has not simplified in the expression for $H_{7}(v)$.

To simplify it, we rewrite it as $^{9}$

$$
\begin{aligned}
H_{8}(v)+\text { c.c. }= & \frac{1}{3} \Delta_{\tau} D_{4}^{(1)}(v)-2 C_{1,1,2}+E_{2}^{2}-E_{4}-2 F_{11}(v) \\
& +\left(H_{3}(v)+\text { c.c. }\right)+\frac{2 \tau_{2}}{\pi} G(v) \partial_{v} G_{2}(v) \bar{\partial}_{v} G_{2}(v)-2\left(H_{9}(v)+\text { c.c. }\right),
\end{aligned}
$$

where the graph

$$
H_{9}(v)=\frac{\tau_{2}^{2}}{\pi^{2}} \int_{w x z} G(v, z) G(v, w) \partial_{x} G(x, z) \partial_{x} G(x) \bar{\partial}_{x} G(w, x) \bar{\partial}_{x} G(v, x)
$$

\footnotetext{
${ }^{9}$ This is obtained by using (A.6) as discussed in the appendix.
} 


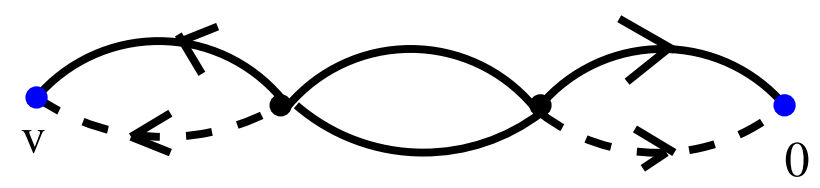

Figure 11. $\pi^{2} \mathcal{K}_{4}(v)$.

depicted by figure 7, is the only term that has not simplified in (3.35) on using (A.4), (A.12) and the expression for $H_{3}(v)$.

Now to simplify $H_{9}(v)$, consider the auxiliary graph

$$
A_{8}(v)=\frac{\tau_{2}^{3}}{\pi^{3}} \int_{w x y z} G(v, z) G(v, y) \partial_{w} G(w, z) \partial_{w} G(w) \bar{\partial}_{x} G(x, y) \bar{\partial}_{x} G(x, v) \bar{\partial}_{w} \partial_{x} G(x, w)
$$

given in figure 8, which leads to

$$
\begin{aligned}
H_{9}(v)= & Q_{1}^{*} Q_{2}(v)+E_{4}+\frac{D_{4}^{(1)}(v)}{2}-\frac{1}{2} E_{2} G_{2}(v)-D_{4}^{(1,2)}(v)-\frac{C_{1,1,2}}{2}-\frac{F_{4}(v)}{2} \\
& +F_{10}(v)-\frac{\tau_{2}}{\pi} \partial_{v} G_{2}(v) \bar{\partial}_{v} G_{3}(v)+\frac{\tau_{2}}{2 \pi} \partial_{v} G_{2}(v) \bar{\partial}_{v} D_{3}^{(1)}(v) .
\end{aligned}
$$

Thus on using (A.4), (A.10) and (A.12) we see $H_{9}(v)$ has simplified.

Hence adding the various contributions, we get that

$$
\begin{aligned}
\mathcal{K}_{3}(v)+c . c .= & \frac{1}{2 \pi} \Delta_{v}\left(G_{2}(v) G_{3}(v)-G_{2}(v) D_{3}^{(1)}(v)-\frac{1}{2} D_{5}^{(1,2,2)}(v)-\frac{1}{6} D_{5}^{(2)}(v)\right) \\
& +\frac{1}{4 \pi} G(v) \Delta_{v} G_{2}(v)^{2}+\frac{1}{3}\left(\Delta_{\tau}-5\right) D_{4}^{(1)}(v)-\frac{1}{2}\left(\Delta_{\tau}-14\right)\left(E_{2}(v) G_{2}(v)\right) \\
& +3 G_{2}(v)^{2}+G(v)\left(4 G_{3}(v)-3 D_{3}^{(1)}(v)+\frac{D_{3}}{3}+\frac{G(v)^{3}}{6}-E_{2} G(v)-2 E_{3}\right) \\
& -\frac{D_{4}}{6}+3 C_{1,1,2}+3 E_{2}^{2}-8 E_{4}+2 D_{4}^{(1,1,2)}(v)-D_{4}^{(2)}(v)+2 G_{4}(v)
\end{aligned}
$$

showing that $\mathcal{K}_{3}(v)+$ c.c. has simplified.

\subsection{Relation involving $\mathcal{K}_{4}(v)$}

We now consider the graph $\mathcal{K}_{4}(v)$ defined by (2.13) which is given by

$$
\mathcal{K}_{4}(v)=\frac{\tau_{2}^{2}}{\pi^{2}} \int_{w z}\left|\partial_{z} G(z, v)\right|^{2}\left|\partial_{w} G(w)\right|^{2} G(z, w)^{2}
$$

as depicted by figure 11 . We rewrite it as

$$
\mathcal{K}_{4}(v)=\frac{\tau_{2}^{2}}{\pi^{2}} \int_{w z}\left|\partial_{z} G(z, v)\right|^{2}\left|\partial_{z} G(z, w)\right|^{2} G(w)^{2}
$$

which we now analyze.

In order to simplify $\mathcal{K}_{4}(v)$, we start with the auxiliary graph

$$
A_{9}(v)=\frac{\tau_{2}^{3}}{\pi^{3}} \int_{w x z} G(x)^{2} \partial_{z} G(z, v) \partial_{z} G(x, z) \bar{\partial}_{w} G(w, v) \bar{\partial}_{w} G(w, x) \bar{\partial}_{z} \partial_{w} G(z, w)
$$


given in figure 8, which leads to

$$
\begin{aligned}
\mathcal{K}_{4}(v)= & 2 E_{2}^{2}+2 D_{4}^{(1,1,2)}(v)-\frac{2 D_{4}^{(1)}(v)}{3}-\frac{2 F_{8}(v)}{3} \\
& +2 F_{11}(v)-\left(F_{6}(v)+\text { c.c. }\right)+2 H_{10}(v)
\end{aligned}
$$

where the graph $H_{10}(v)$ defined by

$$
H_{10}(v)=\frac{\tau_{2}^{2}}{\pi^{2}} \int_{w x z} G(z, v) G(w, v) \partial_{x} G(x, z) \partial_{x} G(x) \bar{\partial}_{x} G(w, x) \bar{\partial}_{x} G(x)
$$

is depicted by figure 7 . Thus from (A.4) we see that $H_{10}(v)$ is the only term which has not simplified in (3.43). To simplify it, we consider the auxiliary graph

$$
A_{10}(v)=\frac{\tau_{2}^{3}}{\pi^{3}} \int_{w x y z} G(z, v) G(w, v) \partial_{x} G(x, z) \partial_{x} G(x) \bar{\partial}_{y} G(w, y) \bar{\partial}_{y} G(y) \bar{\partial}_{x} \partial_{y} G(x, y)
$$

given in figure 8, which leads to

$$
\begin{aligned}
H_{10}(v)= & Q_{2}(v) Q_{2}^{*}(v)+E_{4}+\frac{D_{4}^{(2)}(v)}{2}-\frac{G_{2}(v)^{2}}{2}-2 D_{4}^{(1,2)}(v)-F_{1}(v) \\
& +\left(F_{10}(v)+c . c .\right)-\frac{1}{2}\left(\frac{\tau_{2}}{\pi} \partial_{v} G_{2}(v) \bar{\partial}_{v} D_{3}^{(1)}(v)+c . c .\right)-\left(\frac{\tau_{2}}{\pi} \partial_{v} G_{2}(v) \bar{\partial}_{v} G_{3}(v)+\text { c.c. }\right) .
\end{aligned}
$$

Thus on using (A.4), (A.10) and (A.12), we see that $H_{10}(v)$ has simplified. Hence, adding all the contributions we get that

$$
\begin{aligned}
\mathcal{K}_{4}(v)= & \frac{1}{4 \pi} \Delta_{v}\left(D_{5}^{(2,1,2)}(v)-D_{5}^{(1,2,2)}(v)-\frac{D_{5}^{(2)}(v)}{3}-G_{2}(v) D_{3}^{(1)}(v)-2 G_{2}(v) G_{3}(v)\right) \\
& +\frac{1}{4}\left(\Delta_{\tau}-16\right) G_{2}(v)^{2}+5 E_{4}+2 E_{2}^{2}+3 D_{4}^{(1,1,2)}(v)-D_{4}^{(2)}(v)-2 D_{4}^{(1,2)}(v)-2 G_{4}(v) \\
& +E_{2} G_{2}(v)+G(v)\left(D_{3}^{(1)}(v)-4 G_{3}(v)-G(v) G_{2}(v)-E_{2} G(v)+2 E_{3}\right)
\end{aligned}
$$

Thus $\mathcal{K}_{4}(v)$ has simplified.

Thus we have obtained non-trivial relations involving the elliptic modular graph functions given by (3.19), (3.28), (3.39) and (3.47). In fact, there also exist relations between the graphs whose links are given only by the Green function. For the modular graphs in figure 4 , we have that $[1,8,11,13]$

$$
C_{1,1,2}=\frac{D_{4}}{24}+\frac{3 E_{4}}{4}-\frac{E_{2}^{2}}{8}
$$

while for the various graphs in figures 3 and 4, we also have the relation [36]

$$
D_{4}^{(1,1,2)}(v)-\frac{D_{4}^{(1)}(v)}{6}+\frac{D_{4}^{(2)}(v)}{8}-G_{4}(v)-\frac{G_{2}(v)^{2}}{4}+\frac{1}{2} E_{2} G_{2}(v)+\frac{E_{4}}{4}-\frac{E_{2}^{2}}{8}=0,
$$

which further restrict the relations we have obtained. 


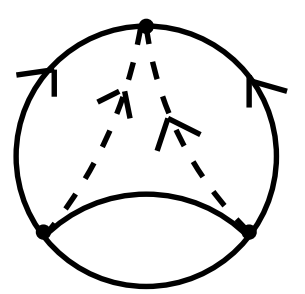

(i)

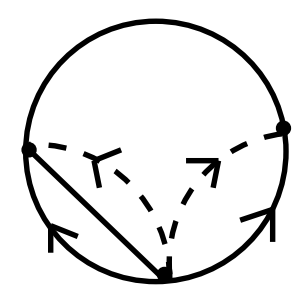

(ii)

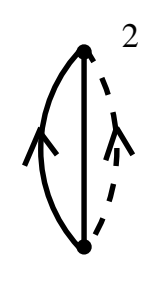

(iii)

Figure 12. (i) $\pi^{2} \mathcal{K}_{5}^{(1)}(v)$, (ii) $\pi^{2} \mathcal{K}_{5}^{(2)}(v)$, (iii) $\pi^{2} \mathcal{K}_{5}^{(3)}(v)$.

\subsection{Relation involving $\mathcal{K}_{5}$}

Finally, we consider $\mathcal{K}_{5}$ defined in (2.14) which we write as

$$
\mathcal{K}_{5}=\mathcal{K}_{5}^{(1)}-2 \mathcal{K}_{5}^{(2)}+\mathcal{K}_{5}^{(3)}
$$

where

$$
\begin{aligned}
\mathcal{K}_{5}^{(1)} & =\frac{\tau_{2}^{2}}{\pi^{2}} \int_{w z}\left|\partial_{z} G(z)\right|^{2}\left|\partial_{w} G(w)\right|^{2} G(z, w)^{2}, \\
\mathcal{K}_{5}^{(2)} & =\frac{\tau_{2}^{2}}{\pi^{2}} \int_{w z}\left|\partial_{z} G(z)\right|^{2}\left|\partial_{w} G(w)\right|^{2} G(z) G(z, w), \\
\mathcal{K}_{5}^{(3)} & =\left[\frac{\tau_{2}}{\pi} \int_{z}\left|\partial_{z} G(z)\right|^{2} G(z)\right]^{2}
\end{aligned}
$$

as depicted by figure 12 .

To simplify $\mathcal{K}_{5}^{(1)}$, we consider the auxiliary graph

$$
A_{11}=\frac{\tau_{2}^{3}}{\pi^{3}} \int_{w x y z} G(x, y)^{2} \partial_{z} G(x, z) \partial_{z} G(y, z) \bar{\partial}_{w} G(w, x) \bar{\partial}_{w} G(w, y) \bar{\partial}_{z} \partial_{w} G(w, z)
$$

given in figure 8 , leading to

$$
\mathcal{K}_{5}^{(1)}=\frac{1}{12} \Delta_{\tau} D_{4}+2 E_{2}^{2}+2 C_{1,1,2}-\frac{5 D_{4}}{6} .
$$

We also have that

$$
\mathcal{K}_{5}^{(2)}=\frac{7 D_{4}}{24}+\frac{C_{1,1,2}}{2}-\frac{E_{2}^{2}}{4},
$$

and

$$
\mathcal{K}_{5}^{(3)}=\frac{E_{2}^{2}}{4} .
$$

Thus we see that $\mathcal{K}_{5}$ has simplified, leading to

$$
\mathcal{K}_{5}=\frac{1}{12}\left(\Delta_{\tau}-\frac{33}{2}\right) D_{4}+\frac{21 E_{2}^{2}}{8}+\frac{3 E_{4}}{4}
$$

on using (3.48). 


\subsection{Some consistency checks}

We now perform some non-trivial consistency checks of the results we have obtained. Consider the case where the two points $v$ and 0 are identified in the graphs $\mathcal{K}_{i}(v)(i=1, \ldots, 4)$. Thus directly from the figures $6,9,10,11$ and 12 , it follows that

$$
\mathcal{K}_{1}(0)=\mathcal{K}_{2}(0)=\mathcal{K}_{3}(0)=\mathcal{K}_{4}(0)=\mathcal{K}_{5}^{(1)}
$$

We now want to check the consistency of (3.57).

First let us consider $\mathcal{K}_{1}(0)$, which from (3.19) yields

$$
\mathcal{K}_{1}(0)=\frac{1}{4}\left(\Delta_{\tau}-4\right) E_{2}^{2}+6 E_{4}-\frac{1}{2} D_{4}-2 C_{1,1,2} .
$$

The equality (3.57) on using (3.53), (3.58) and the constraint (3.48) yields the eigenvalue equation

$$
\left(\Delta_{\tau}-2\right)\left(D_{4}-3 E_{2}^{2}\right)=36 E_{4}-24 E_{2}^{2},
$$

which is precisely the eigenvalue equation $D_{4}$ satisfies $[1,11,13]$.

For the remaining graphs, it is not particularly useful to analyze (3.28), (3.39) and (3.47) directly simply because they have $\Delta_{v}$ acting on various elliptic graphs, and these expressions have to be simplified before the points $v$ and 0 are identified. Thus for these cases, we directly focus on the graphs that arise at intermediate stages of the calculation where the analysis is considerably simpler as the two unintegrated vertices can be identified. Various identities relevant for our analysis are given in (A.5) and (A.11) which we use in the expressions below.

First let us consider $\mathcal{K}_{2}(0)$. Using (3.13), (3.15), (3.18), (3.21), (3.24) and (3.27), we get that

$$
\mathcal{K}_{2}(0)+\mathcal{K}_{5}^{(1)}=\frac{1}{2} \Delta_{\tau} E_{2}^{2}+12 C_{1,1,2}-\frac{5 D_{4}}{3} .
$$

Equating the right hand side to $2 \mathcal{K}_{5}^{(1)}$ and using (3.48) yields (3.59) as the consistency condition.

We next consider $\mathcal{K}_{3}(0)$ and again directly consider the graphs. Using (3.30), (3.33), (3.35) and (3.38), we get that

$$
\mathcal{K}_{3}(0)=\frac{1}{6}\left(\Delta_{\tau}-6\right) D_{4}-\frac{1}{4}\left(\Delta_{\tau}-18\right) E_{2}^{2}-3 E_{4}+2 C_{1,1,2} .
$$

Equating (3.61) to $\mathcal{K}_{5}^{(1)}$ again yields (3.59).

Finally, we consider $\mathcal{K}_{4}(0)$. Now (3.43) and (3.46) leads to

$$
\mathcal{K}_{4}(0)=\frac{1}{4}\left(\Delta_{\tau}-2\right) E_{2}^{2}+3 E_{4}+2 C_{1,1,2}-\frac{2 D_{4}}{3},
$$

which when equated to $\mathcal{K}_{5}^{(1)}$ yields (3.59). Hence we see that the trivial equality of the graphs $\mathcal{K}_{i}(0)(i=1, \ldots, 4)$ with $\mathcal{K}_{5}^{(1)}$ actually yields a non-trivial eigenvalue equation satisfied by the modular graph $D_{4}$. 
Thus we have shown that the graphs $\mathcal{K}_{i}(v)(i=1, \ldots, 5)$ can be expressed in terms of the graphs given in figures 3 and 4 , and contain no additional information. The relations are non-linear in the various graphs, and also involve $\Delta_{\tau}$ and $\Delta_{v}$ acting on some of the graphs in figures 3 and 4 . Note that in each of the equations, the graph involving $\mathcal{K}_{i}(v)$ has two $\partial$ and two $\bar{\partial}$ derivatives and has six links, while each term involving $\Delta_{v}$ contain graphs with a total of five links. Every other term in each equation contains graphs with a total of four links. This is expected, since using (2.4) we see that for the sake of counting the links, removing a $\partial \bar{\partial}$ pair is equivalent to removing a link.

\section{A Some elliptic modular graphs and useful identities}

We now consider the expressions for $F_{i}(v), Q_{1}$ and $Q_{2}(v)$ that arise in the main text. Relevant expressions involving them can be expressed in terms of the graphs given in figures 3 and 4 with significantly less effort than the other graphs in the main text.

We first list the graphs $F_{i}(v)(i=1, \ldots, 11)$ that appear in the main text. They are defined by

$$
\begin{aligned}
F_{1}(v) & =\frac{\tau_{2}}{\pi} \int_{w z} G(z) G(w, v) G(w, z) \partial_{z} G(z, v) \bar{\partial}_{w} G(w), \\
F_{2}(v) & =\frac{\tau_{2}}{\pi} \int_{w z} G(w, v) G(w, z)^{2} \partial_{z} G(z, v) \bar{\partial}_{z} G(z), \\
F_{3}(v) & =\frac{\tau_{2}}{\pi} \int_{w x z} G(w) G(x, v) G(z, v) \partial_{z} G(x, z) \bar{\partial}_{z} G(z, w), \\
F_{4}(v) & =\frac{\tau_{2}}{\pi} \int_{w z} G(z) G(w, v)^{2} \partial_{z} G(v, z) \bar{\partial}_{z} G(w, z), \\
F_{5}(v) & =\frac{\tau_{2}}{\pi} \int_{w z} G(w) G(w, z)^{2} \partial_{z} G(z, v) \bar{\partial}_{w} G(w, v), \\
F_{6}(v) & =\frac{\tau_{2}}{\pi} \int_{w z} G(z)^{2} G(w, v) \partial_{z} G(z, w) \bar{\partial}_{z} G(z, v), \\
F_{7}(v) & =\frac{\tau_{2}}{\pi} \int_{w z} G(z) G(z, v) G(w, v) \partial_{w} G(w, z) \bar{\partial}_{w} G(w), \\
F_{8}(v) & =\frac{\tau_{2}}{\pi} \int_{z} G(z)^{3} \partial_{z} G(z, v) \bar{\partial}_{z} G(z, v), \\
F_{9}(v) & =\frac{\tau_{2}}{\pi} \int_{z} G(z)^{2} \partial_{z} G(z, v) \bar{\partial}_{z} G(z, v), \\
F_{10}(v) & =\frac{\tau_{2}}{\pi} \int_{w x} G(z) G(x, v) G(x, w) \partial_{z} G(z, v) \bar{\partial}_{w} G(w, z), \\
F_{11}(v) & =\frac{\tau_{2}}{\pi} \int_{w x z} G(z) G(w, v) G(x, v) \partial_{z} G(w, z) \bar{\partial}_{z} G(z, x),
\end{aligned}
$$

as depicted by figure 13 .

We also define the graphs $Q_{1}$ and $Q_{2}(v)$ by ${ }^{10}$

$$
\begin{aligned}
Q_{1} & =\frac{\tau_{2}}{\pi} \int_{z_{1} z_{2} z_{3}} \partial_{z_{1}} G\left(z_{1}, z_{2}\right) \partial_{z_{1}} G\left(z_{1}, z_{3}\right) G\left(z_{2}, z_{3}\right), \\
Q_{2}(v) & =\frac{\tau_{2}}{\pi} \int_{z_{1} z_{2}} \partial_{z_{1}} G\left(z_{1}, v\right) \partial_{z_{1}} G\left(z_{1}, z_{2}\right) G\left(z_{2}\right)
\end{aligned}
$$

\footnotetext{
${ }^{10}$ Note that $Q_{2}(0)=Q_{1}$.
} 


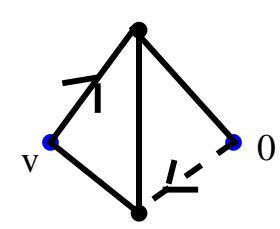

(i)

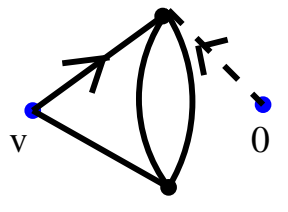

(ii)

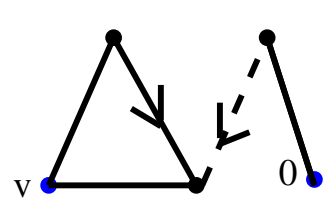

(iii)

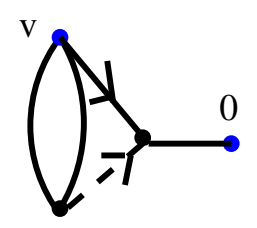

(iv)

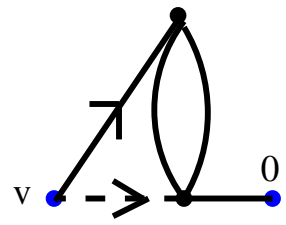

(v)

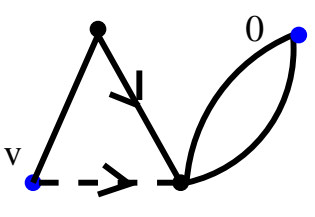

(vi)

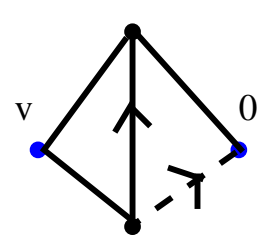

(vii)

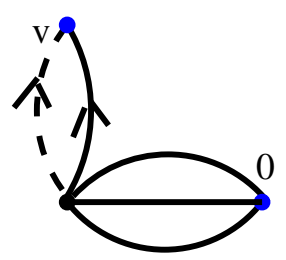

(viii)

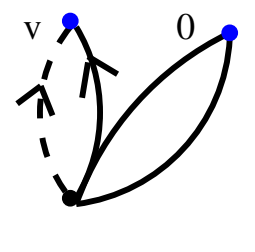

(ix)

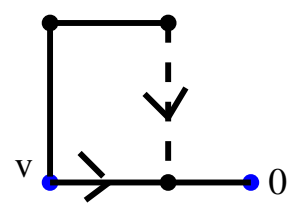

(x)

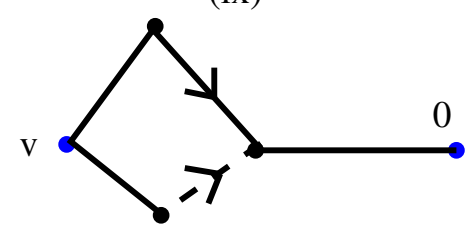

(xi)

Figure 13. (i) $\pi F_{1}(v)$, (ii) $\pi F_{2}(v)$, (iii) $\pi F_{3}(v)$, (iv) $\pi F_{4}(v)$, (v) $\pi F_{5}(v)$, (vi) $\pi F_{6}(v)$, (vii) $\pi F_{7}(v)$, (viii) $\pi F_{8}(v)$, (ix) $\pi F_{9}(v),(\mathrm{x}) \pi F_{10}(v),(\mathrm{xi}) \pi F_{11}(v)$.

which transform as

$$
Q_{1} \rightarrow(c \tau+d)(c \bar{\tau}+d)^{-1} Q_{1}, \quad Q_{2}(v) \rightarrow(c \tau+d)(c \bar{\tau}+d)^{-1} Q_{2}(v)
$$

under $\mathrm{SL}(2, \mathbb{Z})$ transformations.

The graphs (A.1) satisfy useful identities given by

$$
\begin{aligned}
F_{1}(v) & =-G(v) D_{3}^{(1)}(v)+D_{4}^{(1,2)}(v)+\frac{D_{4}^{(2)}(v)}{2}-\frac{G_{2}(v)^{2}}{2}-\frac{1}{8 \pi} \Delta_{v} D_{5}^{(2,1,2)}(v), \\
F_{2}(v)+c . c . & =G(v) D_{3}^{(1)}(v)-C_{1,1,2}+G(v) D_{3}-D_{4}^{(1,1,2)}(v)-D_{4}^{(1)}(v)+E_{2} G_{2}(v), \\
F_{3}(v)+c . c . & =D_{4}^{(1,2)}(v)+D_{4}^{(1,1,2)}(v)+G_{4}(v)-E_{2} G_{2}(v), \\
F_{4}(v)+c . c . & =D_{4}^{(1)}(v)+D_{3} G(v)-D_{4}^{(1,1,2)}(v)-E_{2} G_{2}(v)+C_{1,1,2}-G(v) D_{3}^{(1)}(v), \\
F_{5}(v)+c . c . & =C_{1,1,2}-G(v) D_{3}^{(1)}(v)+D_{4}^{(1)}(v)-E_{2} G_{2}(v)+D_{3} G(v)-D_{4}^{(1,1,2)}(v), \\
F_{6}(v)+c . c . & =E_{2} G(v)^{2}-D_{4}^{(1,1,2)}(v)+D_{4}^{(2)}(v)+\frac{1}{4 \pi} \Delta_{v} D_{5}^{(1,2,2)}(v), \\
F_{7}(v) & =\frac{D_{4}^{(2)}(v)}{2}-\frac{G_{2}(v)^{2}}{2},
\end{aligned}
$$




$$
\begin{aligned}
F_{8}(v) & =-D_{4}^{(1)}(v)+\frac{1}{8 \pi} \Delta_{v} D_{5}^{(2)}(v), \\
F_{9}(v) & =-D_{3}^{(1)}(v)+\frac{1}{8 \pi} \Delta_{v} D_{4}^{(2)}(v), \\
F_{10}(v)+c . c . & =E_{4}-G(v) G_{3}(v)+D_{4}^{(1,2)}(v)+E_{3} G(v)-G_{4}(v), \\
F_{11}(v) & =D_{4}^{(1,2)}(v)+\frac{E_{4}}{2}-\frac{G_{2}(v)^{2}}{2}
\end{aligned}
$$

which we often use. When the vertices at $v$ and 0 are identified, these elliptic modular graphs reduce to modular graphs, hence satisfying several identities, of which the identities

$$
\begin{array}{ll}
F_{1}(0)=\frac{F_{7}(0)}{2}=\frac{1}{4}\left(D_{4}-E_{2}^{2}\right), & F_{2}(0)=\frac{1}{2}\left(E_{2}^{2}-D_{4}\right)-C_{1,1,2}, \\
F_{3}(0)=F_{11}(0)=C_{1,1,2}+\frac{1}{2}\left(E_{4}-E_{2}^{2}\right), & F_{6}(0)=-\frac{4 F_{8}(0)}{3}=\frac{D_{4}}{3}, \quad F_{10}(0)=\frac{C_{1,1,2}}{2}
\end{array}
$$

are used in the main text.

The $\mathrm{SL}(2, \mathbb{Z})$ invariant Laplacian $\Delta_{\tau}$ can be expressed as

$$
\Delta_{\tau}=\bar{\partial}_{\mu} \partial_{\mu}
$$

where $\partial_{\mu}$ is the variation of the Beltrami differential parametrizing the variation of the complex structure modulus on the toroidal worldsheet. In order to obtain the action of $\Delta_{\tau}$ on various graphs using (A.6), we use the relations $[1,9,37,38]$

$$
\partial_{\mu} G\left(z_{1}, z_{2}\right)=-\frac{\tau_{2}}{\pi} \int_{z} \partial_{z} G\left(z, z_{1}\right) \partial_{z} G\left(z, z_{2}\right)
$$

and

$$
\bar{\partial}_{\mu} \partial_{\mu} G\left(z_{1}, z_{2}\right)=0
$$

Along with the eigenvalue equations

$$
\Delta_{\tau} E_{k}=k(k-1) E_{k}, \quad \Delta_{\tau} G_{k}(v)=k(k-1) G_{k}(v),
$$

for $k \geq 2$, we see that the $\mathrm{SL}(2, \mathbb{Z})$ invariant combinations of the graphs appearing in (A.2) yield the relations

$$
\begin{aligned}
Q_{1} Q_{1}^{*} & =\frac{1}{8}\left(\Delta_{\tau}-4\right) E_{2}^{2}, \\
Q_{2}(v) Q_{2}^{*}(v) & =\frac{1}{8}\left(\Delta_{\tau}-4\right) G_{2}(v)^{2}, \\
Q_{1} Q_{2}^{*}(v)+\text { c.c. } & =\frac{1}{4}\left(\Delta_{\tau}-4\right)\left(E_{2} G_{2}(v)\right) .
\end{aligned}
$$

On identifying $v$ and 0 in $Q_{2}(v)$, these yield the relations

$$
Q_{1} Q_{1}^{*}=Q_{2}(0) Q_{2}^{*}(0)=\frac{1}{2}\left(Q_{1}^{*} Q_{2}(0)+\text { c.c. }\right)=\frac{1}{8}\left(\Delta_{\tau}-4\right) E_{2}^{2}
$$

which are used in our analysis. 
Finally, we make use of the identities

$$
\begin{aligned}
\frac{\tau_{2}}{\pi} \partial_{v} G_{2}(v) \bar{\partial}_{v} G_{3}(v)+c . c .= & \frac{1}{4 \pi} \Delta_{v}\left(G_{2}(v) G_{3}(v)\right)+G_{2}(v)^{2}+G(v) G_{3}(v), \\
\frac{\tau_{2}}{\pi} \partial_{v} D_{3}^{(1)}(v) \bar{\partial}_{v} G_{2}(v)+c . c .= & \frac{1}{4 \pi} \Delta_{v}\left(D_{3}^{(1)}(v) G_{2}(v)\right)+G_{2}(v) G(v)^{2} \\
& -E_{2} G_{2}(v)+G(v) D_{3}^{(1)}(v) \\
\frac{\tau_{2}}{\pi} \partial_{v} G_{2}(v) \bar{\partial}_{v} G_{2}(v)= & \frac{1}{8 \pi} \Delta_{v} G_{2}(v)^{2}+G(v) G_{2}(v)
\end{aligned}
$$

to express the graphs with factors of $\partial_{v}$ and $\bar{\partial}_{v}$ along the links in terms of graphs with no derivatives of the Green functions in the links, at the cost of a contribution that has $\Delta_{v}$ acting on it.

Open Access. This article is distributed under the terms of the Creative Commons Attribution License (CC-BY 4.0), which permits any use, distribution and reproduction in any medium, provided the original author(s) and source are credited.

\section{References}

[1] E. D'Hoker, M.B. Green and P. Vanhove, On the modular structure of the genus-one Type II superstring low energy expansion, JHEP 08 (2015) 041 [arXiv: 1502.06698] [INSPIRE].

[2] E. D'Hoker, M.B. Green, O. Gürdogan and P. Vanhove, Modular Graph Functions, Commun. Num. Theor. Phys. 11 (2017) 165 [arXiv:1512.06779] [INSPIRE].

[3] M.B. Green and P. Vanhove, The Low-energy expansion of the one loop type-II superstring amplitude, Phys. Rev. D 61 (2000) 104011 [hep-th/9910056] [INSPIRE].

[4] M.B. Green, J.G. Russo and P. Vanhove, Low energy expansion of the four-particle genus-one amplitude in type-II superstring theory, JHEP 02 (2008) 020 [arXiv:0801.0322] [INSPIRE].

[5] D.M. Richards, The One-Loop Five-Graviton Amplitude and the Effective Action, JHEP 10 (2008) 042 [arXiv: 0807.2421] [inSPIRE].

[6] M.B. Green, C.R. Mafra and O. Schlotterer, Multiparticle one-loop amplitudes and S-duality in closed superstring theory, JHEP 10 (2013) 188 [arXiv:1307.3534] [INSPIRE].

[7] E. D'Hoker and M.B. Green, Exploring transcendentality in superstring amplitudes, JHEP 07 (2019) 149 [arXiv: 1906. 01652] [INSPIRE].

[8] E. D'Hoker, M.B. Green and P. Vanhove, Proof of a modular relation between 1-, 2- and 3-loop Feynman diagrams on a torus, J. Number Theor. 196 (2019) 381 [arXiv:1509.00363] [INSPIRE].

[9] A. Basu, Poisson equation for the Mercedes diagram in string theory at genus one, Class. Quant. Grav. 33 (2016) 055005 [arXiv: 1511.07455] [INSPIRE].

[10] A. Basu, Non-BPS interactions from the type-II one loop four graviton amplitude, Class. Quant. Grav. 33 (2016) 125028 [arXiv:1601.04260] [INSPIRE].

[11] E. D'Hoker and M.B. Green, Identities between Modular Graph Forms, J. Number Theor. 189 (2018) 25 [arXiv:1603.00839] [INSPIRE].

[12] A. Basu, Poisson equation for the three loop ladder diagram in string theory at genus one, Int. J. Mod. Phys. A 31 (2016) 1650169 [arXiv:1606.02203] [INSPIRE]. 
[13] A. Basu, Proving relations between modular graph functions, Class. Quant. Grav. 33 (2016) 235011 [arXiv: 1606.07084] [INSPIRE].

[14] A. Basu, Simplifying the one loop five graviton amplitude in type IIB string theory, Int. J. Mod. Phys. A 32 (2017) 1750074 [arXiv:1608.02056] [INSPIRE].

[15] E. D'Hoker and J. Kaidi, Hierarchy of Modular Graph Identities, JHEP 11 (2016) 051 [arXiv: 1608.04393] [INSPIRE].

[16] A. Kleinschmidt and V. Verschinin, Tetrahedral modular graph functions, JHEP 09 (2017) 155 [arXiv: 1706.01889] [INSPIRE].

[17] A. Basu, Eigenvalue equation for the modular graph $C_{a, b, c, d}$, JHEP 07 (2019) 126 [arXiv: 1906. 02674] [INSPIRE].

[18] J.E. Gerken, A. Kleinschmidt and O. Schlotterer, All-order differential equations for one-loop closed-string integrals and modular graph forms, JHEP 01 (2020) 064 [arXiv:1911.03476] [INSPIRE].

[19] J.E. Gerken, A. Kleinschmidt and O. Schlotterer, Generating series of all modular graph forms from iterated Eisenstein integrals, JHEP 07 (2020) 190 [arXiv:2004.05156] [INSPIRE].

[20] J.E. Gerken, Basis Decompositions and a Mathematica Package for Modular Graph Forms, arXiv:2007.05476 [INSPIRE].

[21] E. D'Hoker, M. Gutperle and D.H. Phong, Two-loop superstrings and S-duality, Nucl. Phys. B 722 (2005) 81 [hep-th/0503180] [INSPIRE].

[22] E. D'Hoker and M.B. Green, Zhang-Kawazumi Invariants and Superstring Amplitudes, arXiv: 1308.4597 [INSPIRE].

[23] E. D'Hoker, M.B. Green, B. Pioline and R. Russo, Matching the $D^{6} R^{4}$ interaction at two-loops, JHEP 01 (2015) 031 [arXiv:1405.6226] [INSPIRE].

[24] B. Pioline, A Theta lift representation for the Kawazumi-Zhang and Faltings invariants of genus-two Riemann surfaces, J. Number Theor. 163 (2016) 520 [arXiv:1504.04182] [INSPIRE].

[25] A. Basu, Perturbative type-II amplitudes for BPS interactions, Class. Quant. Grav. 33 (2016) 045002 [arXiv: 1510.01667] [INSPIRE].

[26] E. D'Hoker, M.B. Green and B. Pioline, Higher genus modular graph functions, string invariants, and their exact asymptotics, Commun. Math. Phys. 366 (2019) 927 [arXiv: 1712.06135] [INSPIRE].

[27] E. D'Hoker, M.B. Green and B. Pioline, Asymptotics of the $D^{8} \mathcal{R}^{4}$ genus-two string invariant, Commun. Num. Theor. Phys. 13 (2019) 351 [arXiv:1806.02691] [InSPIRE].

[28] A. Basu, Eigenvalue equation for genus two modular graphs, JHEP 02 (2019) 046 [arXiv: 1812.00389] [INSPIRE].

[29] E. D'Hoker, C.R. Mafra, B. Pioline and O. Schlotterer, Two-loop superstring five-point amplitudes II: Low energy expansion and S-duality, arXiv:2008.08687 [INSPIRE].

[30] E. D'Hoker and O. Schlotterer, Identities among higher genus modular graph tensors, arXiv:2010.00924 [INSPIRE].

[31] E. D'Hoker and D.H. Phong, Two-loop superstrings VI: Non-renormalization theorems and the 4-point function, Nucl. Phys. B 715 (2005) 3 [hep-th/0501197] [INSPIRE]. 
[32] N. Berkovits, Super-Poincaré covariant two-loop superstring amplitudes, JHEP 01 (2006) 005 [hep-th/0503197] [INSPIRE].

[33] N. Berkovits and C.R. Mafra, Equivalence of two-loop superstring amplitudes in the pure spinor and RNS formalisms, Phys. Rev. Lett. 96 (2006) 011602 [hep-th/0509234] [INSPIRE].

[34] E. D'Hoker, C.R. Mafra, B. Pioline and O. Schlotterer, Two-loop superstring five-point amplitudes. Part I. Construction via chiral splitting and pure spinors, JHEP 08 (2020) 135 [arXiv: 2006. 05270] [INSPIRE].

[35] W. Lerche, B.E.W. Nilsson, A.N. Schellekens and N.P. Warner, Anomaly Cancelling Terms From the Elliptic Genus, Nucl. Phys. B 299 (1988) 91 [InSPIRE].

[36] A. Basu, Poisson equations for elliptic modular graph functions, arXiv:2009.02221 [INSPIRE].

[37] E.P. Verlinde and H.L. Verlinde, Chiral Bosonization, Determinants and the String Partition Function, Nucl. Phys. B 288 (1987) 357 [InSPIRE].

[38] E. D'Hoker and D.H. Phong, The Geometry of String Perturbation Theory, Rev. Mod. Phys. 60 (1988) 917 [INSPIRE]. 